\title{
Electromagnetic form factors of nucleon resonances from CLAS
}

\author{
Evgeny L. Isupov ${ }^{1, *}$, Vitaly $V$. Chesnokov $^{1}$, Evgeny $N$. Golovach ${ }^{1}$, Anna A. Golubenko ${ }^{2}$, \\ Boris $S$. Ishkhanov ${ }^{1,2}$ and Valery A. Klimenko ${ }^{2}$ \\ ${ }^{1}$ Skobeltsyn Institute of Nuclear Physics, Lomonosov Moscow State University, 119234 Moscow, \\ Russia \\ ${ }^{2}$ Department of Physics, Lomonosov Moscow State University, 119991 Moscow, Russia
}

\begin{abstract}
Exclusive single and double meson photo- and electroproduction reactions are the largest sources of information on the spectrum and structure of nucleon resonances. The excited states of the nucleon manifest as a complex interplay between the inner core of three dressed quarks and the external meson-baryon cloud. Various $\mathrm{N}^{*}$ with distinctively different structure appear as unique laboratory where many features of strong QCD can be explored. With combination of nearly $4 \pi$ acceptance of the CLAS detector and continuous electron beam (Jefferson Lab, USA) it is possible to obtain physics observables of the major reaction channels in the $\mathrm{N}^{*}$ excitation region. The results on the electromagnetic transition form factors of $\mathrm{N}(1440) 1 / 2^{+}$and $\mathrm{N}(1520) 3 / 2^{-}$ are presented.
\end{abstract}

\section{Introduction}

Electrons and photons are the best tools to probe the complex internal structure of the nucleon and it's excites states. Exploring the dynamics of nucleon resonances at different scales is crucial to our understanding of quark confinement and the origin of more than $98 \%$ of the visible mass in the Universe.

To study the nonperturbative phenomena such as excitation of nucleon resonances the high-quality data on physics observables in exclusive $\mathrm{N}^{*}$ decay channels are needed. The CLAS detector [1] shown in Fig. 1 provides excellent capabilities for detecting multiparticle final states in photo- and electroproduction processes. The $\mathrm{N}^{*}$ program [2-3] at Jefferson Lab focuses on the exploration of the spectrum of the excited nucleon states as well as determining the electromagnetic transition form factors from the ground nucleon state to the $\mathrm{N}^{*}$ state. The transition form factors depend on the transferred momentum of the virtual photon and encode important pieces of information on how quarks are correlated inside the baryons [4].

Extension to higher photon virtualities will be reached making use of the new CLAS12 detector and upgraded beamline allowing to study the evolution of the strong Quantum Chromodynamics to the regime where perturbative calculations may be relevant.

\footnotetext{
*Corresponding author: isupov@jilab.org
} 


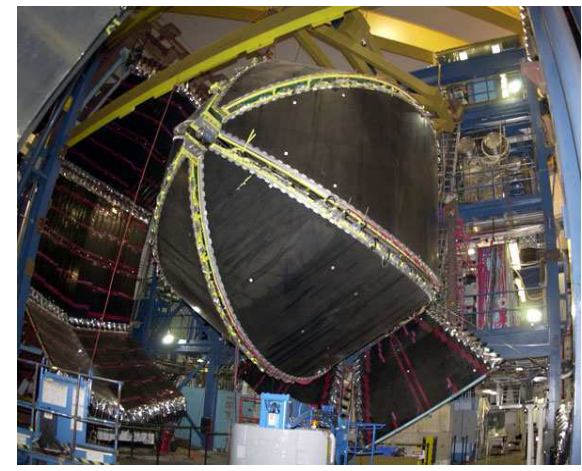

Fig. 1. (Color online) CEBAF Large Acceptance Spectrometer (CLAS).

\section{Data Analysis}

Majority of the physics observables pertaining to the $\mathrm{N}^{*}$ excitation region in single and double meson production were measured in CLAS, see Ref. [5] for details. The CLAS Physics Database [6] contains data files with all measured observables. In order to extract the electromagnetic transition form factors from the experimental data the reaction models need to be built. For the single pion/eta electroproduction the approach based on fixed- $t$ dispersion relations (DR) [7] as well as the effective Lagrangian approach - Unitary Isobar Model (UIM) [8] were developed. The quality of the DR and UIM data fits and sensitivity of the dispersion relations approach to the $\mathrm{N}(1440) 1 / 2^{+}$contribution are shown in Fig. 2.



Fig. 2. (Color online) Single pion electroproduction $\gamma^{v} \mathrm{p} \rightarrow \mathrm{n} \pi^{+}$cross sections [9] for $\mathrm{Q}^{2}=2.05 \mathrm{GeV}^{2}$ with model fits [10] (red solid line - DR, blue solid line - UIM, red dotted - DR without N(1440)1/2+ contribution).

For the double charged pion production, the phenomenological isobar model (JM) [11] with unitarized Breit-Wigner ansatz was constructed. Due to high statistics it was possible to extract nine one-fold differential cross sections for the reaction $\gamma^{\mathrm{r}, \mathrm{v}} \mathrm{p} \rightarrow \mathrm{p} \pi^{+} \pi^{-}[12-17]$. The quality of the data allowed for determining all essential mechanisms contributing to the two pion photo- and electroproduction cross sections. The examples of data description by JM model for the two pion photoproduction data [12] at $\mathrm{W}=1.74 \mathrm{GeV}$ is given in Fig. 3 and for the electroproduction data [16-17] at $\mathrm{W}=1.71 \mathrm{GeV}, \mathrm{Q}^{2}=2.6 \mathrm{GeV}^{2}$ in Fig. 4. The phenomenological models rely on the experimental data therefore their scope is limited by 
the phase-space available in the current experiments. Because the non-resonant mechanisms in different exclusive channels are completely unalike, consistent results on electrocouplings give additional credibility of the extraction procedures.
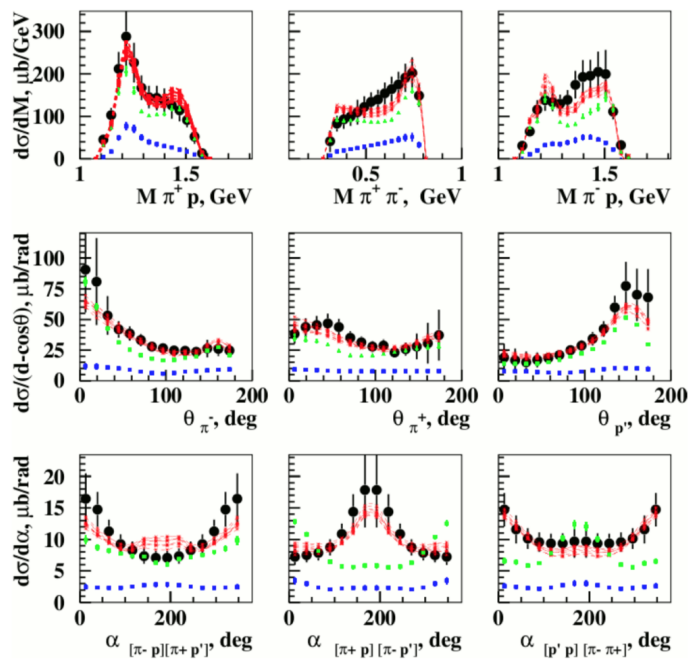

Fig. 3. (Color online) Two pion photoproduction 1-fold differential cross sections (black circles) for $\mathrm{W}=1.74 \mathrm{GeV}$ and the resonant/non-resonant contributions (blue/green points) from the JM model fits (red curve).

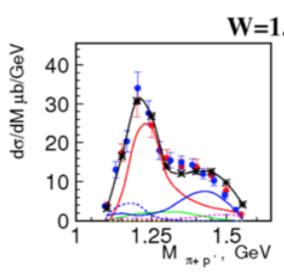

$\mathrm{W}=1.71 \mathrm{GeV}, Q^{2}=2.6 \mathrm{GeV}^{2}$
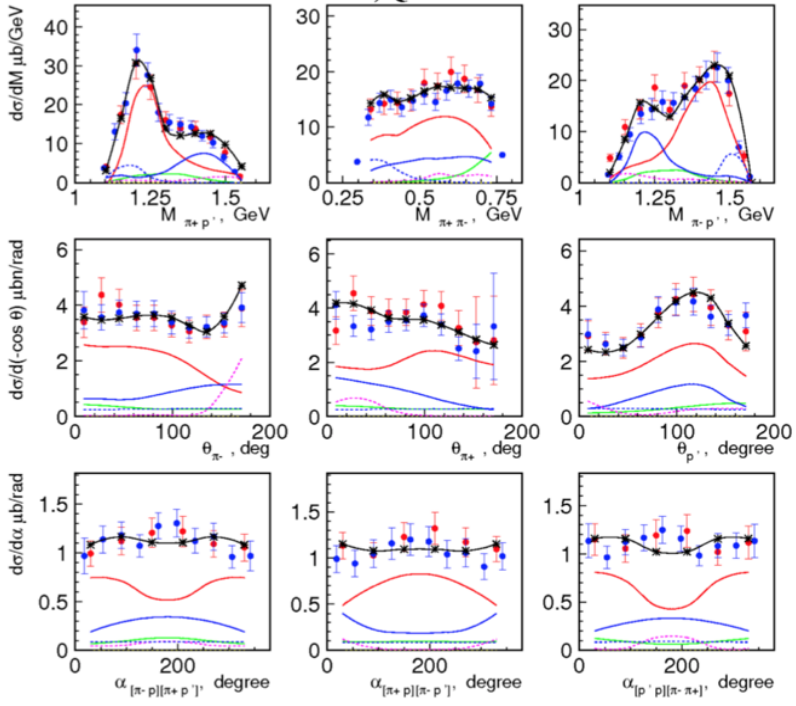

Fig. 4. (Color online) Two pion electroproduction 1 -fold differential cross sections for $\mathrm{W}=1.71$ $\mathrm{GeV}, \mathrm{Q}^{2}=2.6 \mathrm{GeV}^{2}$ with JM model fits (black solid line).

The results for the transition amplitudes $A_{1 / 2}$ and $S_{1 / 2}$ of the Roper $\left(N(1440) 1 / 2^{+}\right)$ resonance extracted from the single and double (preliminary) pion production are shown in Fig. 5 [3]. The behaviour of the amplitudes rules out the hybrid hypothesis for the Roper. The detailed analysis is given in Ref. [18]. 
The form factors $A_{1 / 2}, A_{3 / 2}$ and $S_{1 / 2}$ of $N(1520) 3 / 2^{-}$are shown in Fig. 6 [3]. The data confirm dominance of $\mathrm{A}_{3 / 2}$ amplitude at the photon point with subsequent shift at $\mathrm{Q}^{2}>0.5$ $\mathrm{GeV}^{2}$ to the $\mathrm{A}_{1 / 2}$ dominance. The electrocouplings of other resonances extracted from the CLAS data can be found in Ref. [19].
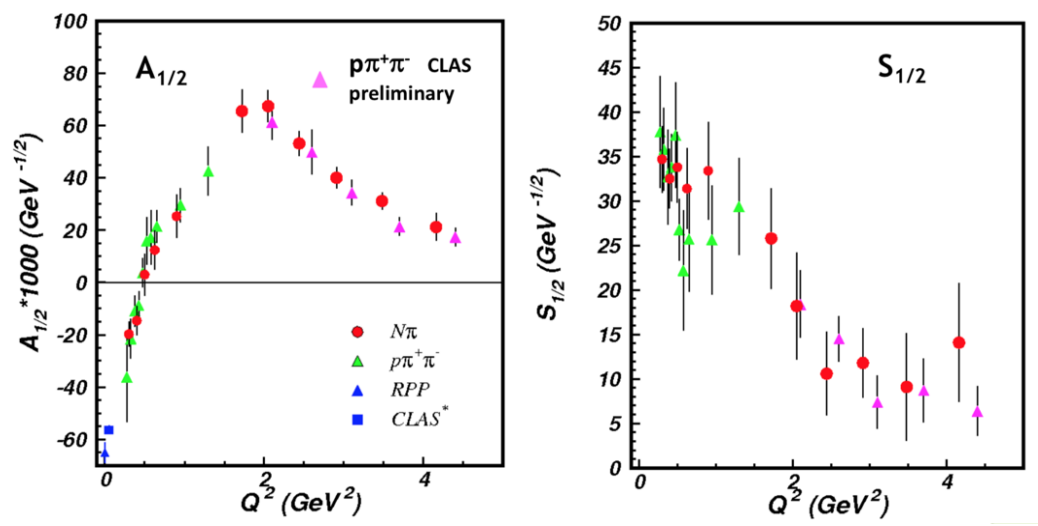

Fig. 5. (Color online) Electromagnetic transition form factors $A_{1 / 2}$ and $S_{1 / 2}$ of the Roper resonance.
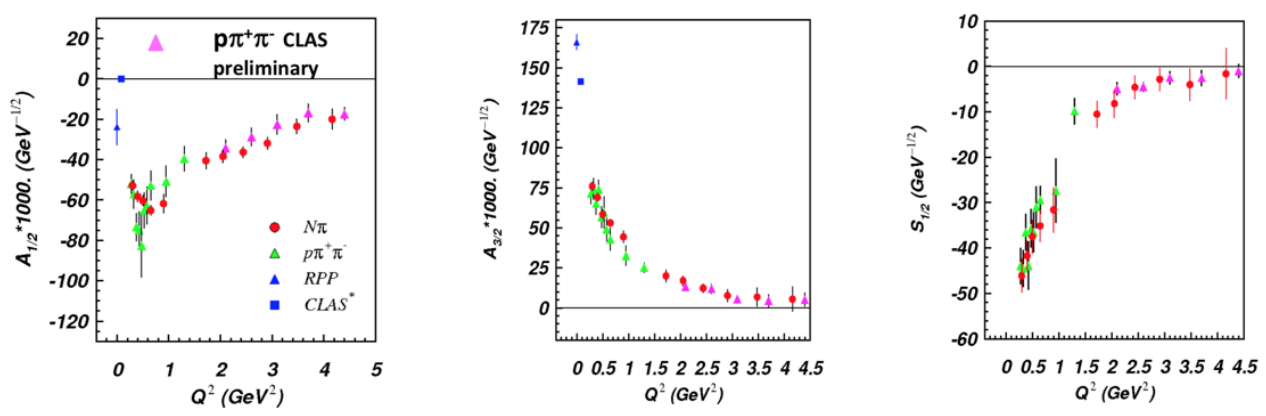

Fig. 6. (Color online) Electromagnetic transition form factors $A_{1 / 2}, A_{3 / 2}$ and $S_{1 / 2}$ of $N(1520) 3 / 2$.

\section{Conclusions}

The extensive research program of nucleons resonances spectrum and structure is ongoing in Jefferson Lab. Electromagnetic transition form factors of the most nucleon resonances have become available in the region of photon virtualities $0-5 \mathrm{GeV}^{2}$. We observe consistent results on resonance photo- and electrocouplings extracted from the single pion/eta production channels and the reaction of double pion production.

The analysis based on the Schwinger-Dyson equations (DSE) in QCD [20-21] has been shown effective in revealing the complex dynamics of quarks and gluons leading to significant effects of diquark correlations and quark exchanges in baryons. Within the DSE approach the momentum-dependent dressed quark mass function was calculated [22] and the elastic nucleon form factors, $\mathrm{p}-\mathrm{N}(1232) 3 / 2^{+}$and $\mathrm{p}-\mathrm{N}(1440) 1 / 2^{+}$transition form factors were described simultaneously using exactly the same mass function.

The data on $\mathrm{p} \pi^{+} \pi^{-}$photo- and electroproduction will allow us determine the electromagnetic transition amplitudes of almost all nucleon resonances in the mass range from $1.6 \mathrm{GeV}$ to $2.0 \mathrm{GeV}[3]$. 
The information on $\mathrm{N}^{*}$ electrocouplings is essential for understanding the mechanisms of hadron mass generation and the nature of confinement of quarks and gluons in QCD.

This work was supported by the U.S. Department of Energy, Office of Science, Office of Nuclear Physics under contract DE-AC05-06OR23177.

\section{References}

1. B.A. Mecking et al., Nucl. Inst. Meth. A 503, 513 (2003)

2. V.D. Burkert The 12th International Workshop on the Physics of Excited Nucleons $N^{*} 2019$, (to be published)

3. V.I. Mokeev The 12th International Workshop on the Physics of Excited Nucleons $N^{*} 2019$, (to be published)

4. C.D. Roberts The 12th International Workshop on the Physics of Excited Nucleons $N^{*} 2019$, (to be published)

5. I.G. Aznauryan, and V.D. Burkert, Prog. Part. Nucl. Phys. 1 67, (2012)

6. CLAS physics database, http://clasweb.jlab.org/physicsdb

7. I.G. Aznauryan et al., Phys. Rev. C 80, 055203 (2009)

8. I.G. Aznauryan Phys. Rev. C 67, 015209 (2003)

9. K. Park et al. (CLAS Collaboration), Phys. Rev. C 77, 015208 (2008)

10. I.G. Aznauryan et al., Phys. Rev. C 78, 045209 (2008)

11. V.I. Mokeev et al., Phys. Rev. C 93, 025206 (2016)

12. E.N. Golovatch et al., CLAS Collaboration, Phys. Lett. B 371 788, (2019)

13. M. Ripani et al., CLAS Collaboration, Phys. Rev. Lett. 022002 91, (2003)

14. G.V. Fedotov et al., CLAS Collaboration, Phys. Rev. 015204 C 79, (2009)

15. E.L. Isupov et al., CLAS Collaboration, Phys. Rev. 025209 C 96, (2017)

16. A. Trivedi, Few Body Syst. 45 60, (2019)

17. G.V. Fedotov et al., CLAS Collaboration, Phys. Rev. 025203 C 98, (2018)

18. V.D. Burkert and C.D. Roberts, Rev. Mod. Phys. 011003 91, (2019)

19. A.N. Hiller Blin et al., Phys. Rev. 035201 C 100, (2019)

20. J. Segovia et al., Phys. Rev. Lett. 171801 115, (2015)

21. J. Segovia, Few Body Syst. 1185 55. (204)

22. D. Binosi et al., Phys. Rev. D 95, 031501 (2017) 